\title{
Zebrafish fin regeneration involves transient serotonin synthesis
}

\author{
Désirée König; Anna Jaźwińska \\ Department of Biology, University of Fribourg, Chemin du Musée 10, 1700, Fribourg, Switzerland
}

\author{
Reprint requests: \\ Anna Jaźwińska, Department of Biology, \\ University of Fribourg, Chemin du Musée \\ 10, 1700 Fribourg, Switzerland. \\ Tel: +41263008890; \\ Email: anna.jazwinska@unifr.ch
}

\begin{abstract}
The zebrafish is a vertebrate organism capable of regenerating many of its organs. Notably, it can undergo epimorphic regeneration of its fins after amputation. This process occurs through the formation of a wound epithelium and the dedifferentiation of mesenchymal and bone-forming cells, which form a proliferative blastema. Here, we report that the entry into the regenerative process involves the local synthesis of serotonin (5-hydroxytryptamine, 5-HT) in the injury-associated tissue. One day after wounding, intracellular accumulation of serotonin was induced in the stump below the amputation plane. During blastema formation, serotonin was detected in the mesenchyme at the vicinity of the amputation plane and in the apical wound epithelium. During the advanced outgrowth phase, this monoamine was no longer present in the blastema, suggesting a temporal involvement of serotonin in the postinjury area. We show the expression of two serotonin synthesizing enzymes, tryptophan hydroxylase $l a$ and $l b$ in the blastema, suggesting the local production of this monoamine. Neither depletion of serotonin by chemical inhibition of tryptophan hydroxylase, nor ectopic administration of this monoamine affected fin regeneration, indicating it does not play a role during this process. Finally, we found that the presence of serotonin during regeneration depends on fibroblast growth factor and retinoic acid signaling. Overall, our study demonstrates that the initiation of fin regeneration is associated with a transient synthesis of serotonin in the regrowing tissue.
\end{abstract}

\section{INTRODUCTION}

The zebrafish fin is a dermal appendage that is able to completely regrow after amputation through a process called epimorphic regeneration. This mechanism relies on the capacity of the stump to reactivate morphogenetic programs that manifest in the formation of a wound epithelium and a blastema within two days after injury. ${ }^{1,2}$ Importantly, both these structures originate from the mobilized stump tissues. The wound epidermis derives from migrating epithelial cells at the site of injury, while the blastema arises by the conversion of local non-dividing cells into lineage-restricted proliferating cells, which give rise to the outgrowth. $^{3-5}$ The blastema comprises mesenchymal cells and osteoblasts, both of which are components of connective tissue in the fin. The distal-most part of the regenerative outgrowth is thought to act as an organizer through its signaling activity, whereas the proximal blastema comprises the proliferation and re-differentiation zone. ${ }^{1,6}$ A combination of signaling pathways, such as FGF, Wnt, TGFß/Activin, BMP, IGF, Notch, Shh, retinoic acid, and Hippo, regulate the regenerative process. ${ }^{7}$ In addition, systemic factors, such as innervation and immune response, contribute to efficient tissue regrowth. ${ }^{8,9}$ The involvement of specific neurotransmitters in the context of fin regeneration has not yet been systematically investigated.

Serotonin (5-hydroxytryptamine; 5-HT) is a monoamine neurotransmitter, which plays a role in the regulation of neuropsychological activities, such as mood, perception, reward, memory, appetite, sleep, sexuality, pain perception, and social behaviors. ${ }^{10}$ Serotonin is synthesized in a two-step reaction from tryptophan not only in neurons but also in several other tissues, such as in intestinal cells. ${ }^{11}$ The monoamine is distributed by blood platelets throughout the entire body, where it modulates diverse non-neural processes, including various metabolic roles, vascular constriction, platelet aggregation, inflammation, insulin release, intestinal motility, and bladder control. ${ }^{12-14}$

Behavioral studies in zebrafish have demonstrated the conservation among vertebrates of the different neurological functions of serotonin, as well as of the effects of drugs, which affect the serotonergic system. ${ }^{15}$ In zebrafish peripheral organs, serotonin was detected in Merkel-like cells of the taste buds, in neuroepithelial cells of the skin, and joint-associated fibroblasts in the fin. ${ }^{16-19}$ However, the complexity of serotonin distribution in epidermal and blastemal cells during fin regeneration remains elusive. In this study, we aimed to investigate the dynamics of serotonin synthesis and the function of this monoamine during fin regeneration. Finally, we addressed the requirement of serotonin for fin regeneration and the mechanisms regulating its deposition in the fin outgrowth.

\section{MATERIALS AND METHODS}

\section{Fish strains and fin amputations}

For this study, the following strains were used: $A B$ strain (Oregon), hsp70:dnfgfrl-egfp, ${ }^{20}$ dob (devoid of blastema, $\left.f g f 20 a^{--}\right) .^{21}$ Adult fish were used at ages 12-24 months. For heat-shock experiments, the animals were placed in a 
water bath regulated by a thermostat equipped with a cooling system (Lauda Eco, Lauda-Königshofen, Germany), which was programmed to increase the temperature to $37^{\circ} \mathrm{C}$ for 1 hour and cool back down. For fin amputation, fish were anesthetized in $0.6 \mathrm{mM}$ tricaine (MS-222 ethyl-m-aminobenzoate, SigmaAldrich, Saint-Louis, Missouri, United-States), and fins were
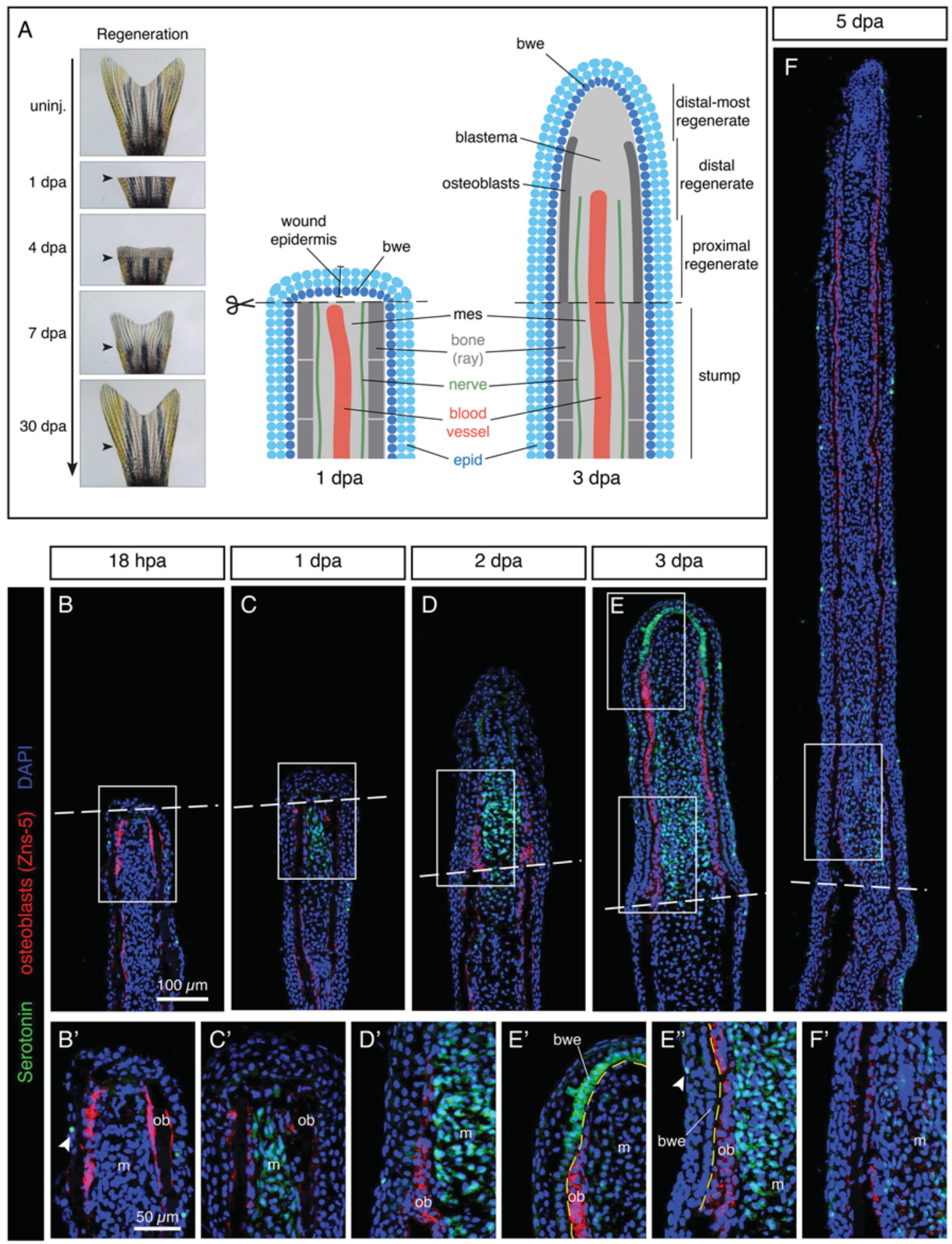

Figure 1. Legend on next page. 
amputated with a razor blade proximal to the first bone bifurcation point. Animals were kept at $27{ }^{\circ} \mathrm{C}$ for various durations before fin collection. Live images of regenerating fins were taken with a Leica AF M205 FA stereomicroscope (Leica Microsystems, Wetzlar, Germany). Animal experimentation was performed in accordance with Swiss regulations and approved by the Cantonal Veterinary office of Fribourg, Switzerland.

\section{Drug treatments}

Drug treatments were performed in $100 \mathrm{~mL}$ Coplin jars (up to 3 fish per Coplin jar). The following drugs were used: para-chlorophenylalanine (pcpa, 2.5-10 mM; Sigma-Aldrich, Saint-Louis, Missouri, United-States), BrdU (163 $\mu \mathrm{M}=$ $50 \mathrm{mg} / \mathrm{L}$; Sigma-Aldrich, Saint-Louis, Missouri, United-States), SB431542 (10 $\mu \mathrm{M}$; Tocris, Avonmouth, Bristol, United Kingdom), Retinoic acid (1 $\mu \mathrm{M}$; Sigma-Aldrich, Saint-Louis, Missouri, United-States). Drugs were dissolved in DMSO or water depending on polarity and mixed into fish water. Control fish were kept in $100 \mathrm{~mL}$ Coplin jars containing 0.1\% DMSO when appropriate.

\section{Injection of serotonin}

Serotonin hydrochloride (Sigma-Aldrich, Saint-Louis, Missouri, United-States) was dissolved to $50 \mathrm{mM}$ in Hank's balanced salt solution (Thermo Fisher, Waltham, Massachusetts, UnitedStates). Phenol red was added to the mix to allow visualization of the injection. Fish were anesthetized and serotonin solution was injected (Eppendorf Femtojet microinjector, Eppendorf, Hamburg, Germany) into every ray blastema of the ventral lobe of the caudal fin at $2 \mathrm{dpa}$. Control fish were injected with Hank's balanced salt solution in the ray blastemas of the ventral lobe. About $1 \mu \mathrm{L}$ of the solution was injected in every fin. Images of regenerating fins were taken on subsequent days depending on experimental design.

\section{Immunofluorescence staining of fin sections}

Fins were harvested at different timepoints after amputation, fixed in $2 \%$ paraformaldehyde overnight at $4{ }^{\circ} \mathrm{C}$ and embedded in tissue freezing medium. Sections were cut to $16 \mu \mathrm{m}$ thickness. Immunofluorescence stainings were performed as previously described. ${ }^{22}$ Briefly, the slides were blocked for 1 hour in blocking solution (5\% goat serum (Jackson Immunoresearch, West Grove, Pennsylvania, United-States) in $0.3 \%$ Triton-X in PBS (PBST)), incubated overnight in primary antibodies diluted in blocking solution, washed on the next day in PBST, incubated in secondary antibody for 2 hours, washed and mounted in $90 \%$ glycerol in $20 \mathrm{mM}$ Tris $\mathrm{pH} 8$ with $0.5 \%$ N-propyl gallate (Sigma-Aldrich, Saint-Louis, Missouri, United-States). For BrdU visualization, the sections were incubated for 40 minutes in a solution of $2 \mathrm{~N} \mathrm{HCl}$ before immunofluorescence staining.

The following primary antibodies were used: Rabbit antiSerotonin (1:1000, Sigma-Aldrich, Saint-Louis, Missouri, United-States), Mouse Zns5 (1:250, Zebrafish International Resource Center, Eugene, Oregon, United-States), Rat antiBrdU (1:200, Abcam). Fluorescent dye-coupled secondary antibodies (Jackson Immunoresearch, West Grove, Pennsylvania, United-States) were used at 1:500. DAPI (SigmaAldrich, Saint-Louis, Missouri, United-States) was used to label nuclei. Immunofluorescence was imaged using a Leica SP5 confocal microscope (Leica Microsystems, Wetzlar, Germany).

\section{Image processing and analysis}

Images from confocal microscopy were processed using Image J (NIH, Bethesda, Maryland, United-States) and Photoshop (Adobe, San Jose, California, United-States).

Serotonin-positive domain positions were measured on longitudinal sections by tracing and measuring lines from the amputation plane (0) to the proximal and distal edge of the serotonin-positive domains of the mesenchyme. Any position proximal to the amputation plane was a negative number and a position distal to the amputation plane was a positive number.

Length of regenerates was measured on the live images by tracing and measuring lines from the amputation plane to the tip of the ray on the 2 nd to 4th rays from the lateral edge (longest rays in the fin).

The effects of serotonin injections were evaluated by measuring the areas of the ventral and dorsal fin lobes beyond the amputation plane in the live images of control injected and serotonin injected fins. For each fin, the ratio of ventral (injected) fin lobe over dorsal (uninjected) fin lobe was calculated.

Graphs were plotted in Graphpad (Graphpad, San Diego, California, United-States) prism or Microsoft Excel (Microsoft, Redmond, Washington, United-States). Student $T$-tests were performed comparing experimental groups to control

Figure 1. Serotonin accumulates in the proximal mesenchyme and distal-most basal wound epidermis of regenerating fins. (A) Schematic representation of fin regeneration. Live imaging of a regenerating fin (left panel) and schematic representations of longitudinal sections through rays at 1 and $3 \mathrm{dpa}$ (right panel). At $1 \mathrm{dpa}$, the injury plane is covered by a wound epidermis. The basal layer of the wound epidermis (bwe, dark blue) provides signals to the underlying mesenchyme (mes, light gray). At 3 dpa, a regenerative blastema is formed with proliferating cells. Nerves, blood vessels, and osteoblasts grow into the new tissue. (BF) Immunofluorescence stainings for serotonin and Zns-5 (osteoblasts, ob) on longitudinal sections of $18 \mathrm{hpa}, 1 \mathrm{dpa}, 2 \mathrm{dpa}$, $3 \mathrm{dpa}$, and $5 \mathrm{dpa}$ fins. No serotonin is present in the mesenchyme at $18 \mathrm{hpa}\left(\mathrm{B}^{\mathrm{B}} \mathrm{B}^{\prime}\right)$. Serotonin is found in the dedifferentiating mesenchyme $(\mathrm{m})$ at $1 \mathrm{dpa}\left(\mathrm{C}-\mathrm{C}^{\prime}\right)$ and in the proximal mesenchyme during blastema formation and early regenerative outgrowth $\left(D-E^{\prime \prime}\right)$. In addition, serotonin is detected in the basal wound epidermis (bwe) of the distal-most blastema at 3 dpa ( $\left.E, E^{\prime}\right)$. Yellow dashed line highlights the basal membrane. By $5 \mathrm{dpa}$, serotonin immunofluorescence is weaker but still visible near the amputation plane $\left(F-F^{\prime}\right)$. White dashed lines highlight amputation planes (This applies to all subsequent figures). $\mathrm{N} \geq 4$ for each time point. $\left(B^{\prime}, C^{\prime}, D^{\prime}, E^{\prime}, F^{\prime}\right)$ Higher magnifications of the framed area shown in the images that are labeled with the same letter but without the prime symbol (The same rule applies to all the subsequent figures). Arrowheads indicate sporadic serotonin-positive cells in the epidermis. 
groups. Unless indicated otherwise in figure legends, error bars indicate standard error.

\section{In situ hybridization}

In situ hybridization on sections was performed as previously described. ${ }^{22}$

Primers for the generation of the template for the probes were the following:

tphla (NM_178306.3): Fw 5'-aagcgagatggagaatgtgc-3'; Rev: 5'-tgcatctccaagatgtccag-3'.
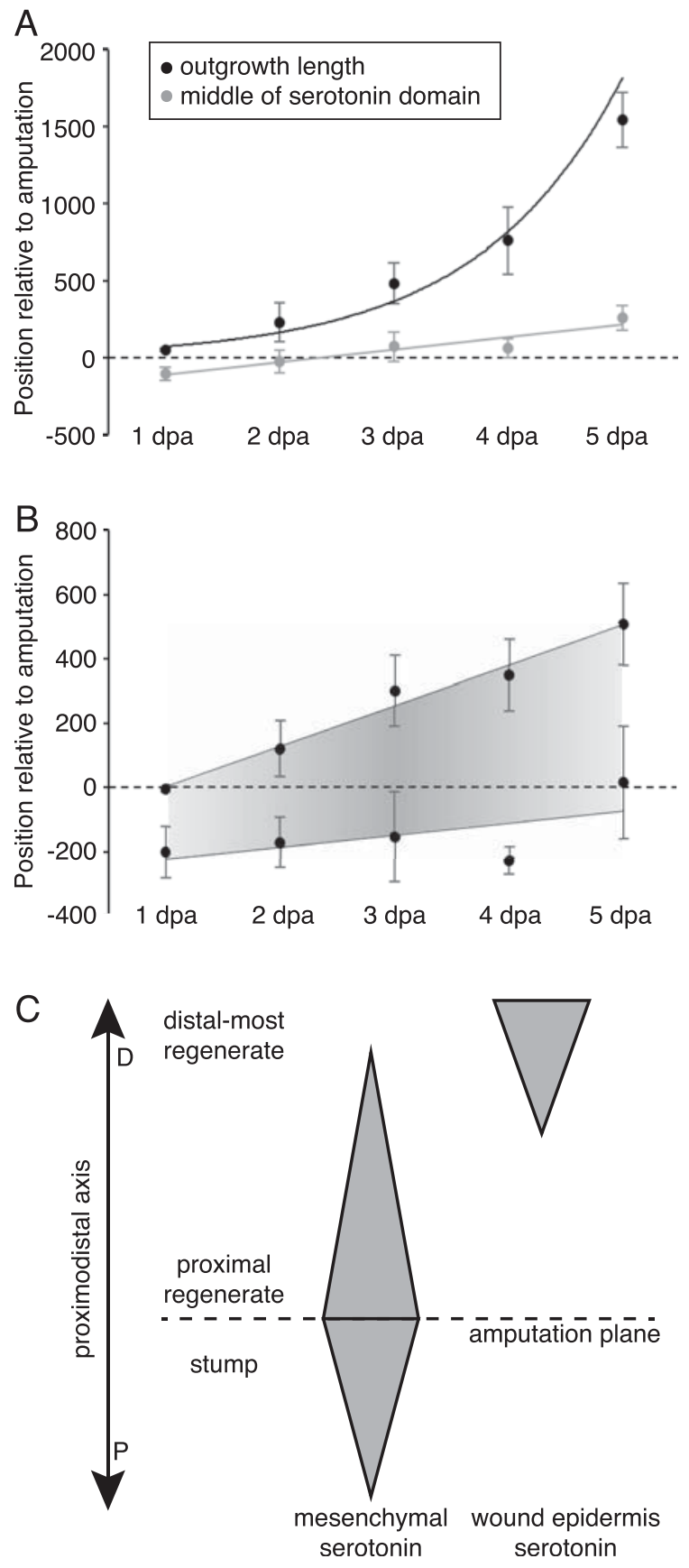

tphlb (NM_001001843.2): FW 5'-gggctggtcttctctcttcc-3'; Rev: $5^{\prime}$-cagctcatggcaagaaacag- $3^{\prime}$

tph2 (NM_001310068.1): Fw 5'-tctcagagctggatcagtgc-3'; Rev: 5'-tcacagacggtggttagtcg- $3^{\prime}$.

mao (NM_212827): FW 5'-agaccaaatgggtggatctg-3'; Rev: $5^{\prime}$-ctcctctgatcccaggacac- $3^{\prime}$.

\section{Quantitative real-time polymerase chain reaction}

For cDNA generation, fin tissue was collected at $0 \mathrm{dpa}$ (a piece of tissue from the fin, not including the distal edge of the fin) and $3 \mathrm{dpa}$ (regenerate only), and frozen on dry ice. For each time point, two cDNAs were generated. For each cDNA, the pooled tissue from 10 fish was used. The collected tissue was homogenized in Qiazol Lysis Reagent (Qiagen, Hilden, Germany) using a Polytron tissue homogenizer. RNA was extracted using chloroform and isolated from tissue debris using MaXtract high density columns (Qiagen, Hilden, Germany). RNA was precipitated using isopropanol and resuspended in water. Reverse transcription was performed to obtain cDNA using the Superscript III reverse transcriptase (Thermo Fishers, Waltham, Massachusetts, United-States), following the manufacturer's protocol.

qRT-PCR was performed using the Kapa SYBR Fast qPCR kit (Kapa Biosystems, Wilmington, Massachusetts, United-States) following the manufacturer's guidelines. The following primers were used:

tphla (NM_178306.3): Fw 5'-agtcggtgggctagtcaagg-3'; Rev: 5'-agatttccgggactcaatgtg- $3^{\prime}$.

tphlb (NM_001001843): Fw 5'-gagctttgaggaagccaaatg-3';

Rev: $5^{\prime}$-gagagaatggacgctggattg- $3^{\prime}$.

tph2 (NM_001310068.1): FW 5'-ctttctggtgaaagcgctgag-3'; Rev: 5'-tcaatgtgcgccaaattcac-3'.

$\beta$-actin 2 (NM_181601.5): Fw 5'-ttggcaatgagaggttcagg-3'; Rev: 5'-tggagttgaaggtggtctcg-3'.

pabpcla (NM_001031676.1): Fw 5'-ttggtttcgtgagcttcgag3'; Rev: 5'-acctgcttccegttcatctc-3'.

Normalized gene expression was calculated using the Pfaffl method. Gene expression levels were averaged over two cDNAs per time point and three qRT-PCR reactions. Results were plotted in Graphpad Prism (Graphpad, San Diego, California, United-States)

Figure 2. Serotonin-positive regions in the mesenchyme (A) Localization of the middle of the serotonin-positive mesenchymal domain (gray points and line) at different time points of regeneration compared to the length of the regenerative outgrowth (black points and line). Zero on the $y$-axis represents the amputation plane, negative numbers are proximal to the amputation plane, and positive numbers are distal to the amputation. Distances on Y-axis indicated in $\mu \mathrm{m}$. Error bars are standard deviation. $N \geq 4$ for each time point. (B) Localization of the distal and proximal edge of the serotonin-positive mesenchymal domain at different time points of regeneration. The domains overlap the amputation plane at all time points. Distances on Y-axis indicated in $\mu \mathrm{m}$. Error bars are standard deviation. $\mathrm{N} \geq 4$ for each time point. (C) Schematic illustration of the intensity of serotonin immunolabeling in mesenchymal cells vs. basal wound epidermal cells on the proximodistal axis during outgrowth formation at $3 \mathrm{dpa}$. Concentration gradients are inverted in the two tissues. 


\section{RESULTS}

\section{Transient presence of serotonin in non-neural tissues during the formation of a regenerative outgrowth}

Regeneration of the fin occurs through the formation of a specialized wound epidermis and a proliferative blastema within the first day after amputation ${ }^{6,7}$ (Figure 1A). Serotonin has previously been detected in the regenerative outgrowth ${ }^{19}$ however, its dynamics during regeneration are not completely characterized. To investigate the distribution of serotonin at different stages of regeneration, we collected fins at subsequent days after amputation and immunostained sections with a validated rabbit serotonin antibody. ${ }^{23}$ Rays were identified with a mouse Zns-5 antibody, which detects bone-forming osteoblasts. ${ }^{24}$ During wound healing, up to 18 hours post-amputation (hpa), no serotonin expression was detected in the mesenchyme of the stump (Figure 1B). However, during blastema formation, at 1 day postamputation (dpa), mesenchyme within a distance of $200 \mu \mathrm{m}$ below the amputation plane displayed serotonin immunoreactivity (Figure 1C). At 2 dpa, the serotonin immunolabeled mesenchyme expanded to approximately $200 \mu \mathrm{m}$ above the amputation plane, without reaching the distal-most blastema (Figure 1D). At 3 and $4 \mathrm{dpa}$, serotonin persisted at a similar position in the mesenchyme relative to the amputation plane, despite a substantial elongation of the regenerative outgrowth (Figure 1E). By $5 \mathrm{dpa}$, serotonin immunoreactivity became weaker, still remaining at the vicinity of the amputation plane (Figure 1F). Starting from $6 \mathrm{dpa}$, serotonin was absent from the mesenchyme. We concluded that serotonin accumulates transiently during a few days after wound healing and initiation of regeneration.

To further characterize the presence of serotonin during early regeneration, we analyzed the median position and the proximal and distal ends of the serotonin-positive mesenchymal tissue relative to the amputation plane and the regenerative outgrowth (Figure 2A and B). While the regenerative outgrowth progresses between 1 and $5 \mathrm{dpa}$, the serotoninpositive domain remains at the vicinity of the amputation plane (Figure 2A). Measurements of the proximal and distal end of the serotonin-positive domain at these time points show that the domain constantly overlaps the amputation plane (Figure 2B). Thus, serotonin in the mesenchymal tissue provides a transient marker of the injury site.

In addition to the mesenchymal serotonin, we observed the presence of serotonin in the distal-most basal wound epidermis on sections from 2 to 4 dpa (Figure 1E and E'). Interestingly, the transition between high- and low-serotonin staining of the wound epithelium coincided with the margin of migrating Zns5-labeled osteoblasts (Figure 1E'). Notably, all osteoblasts were clearly negative for serotonin immunoreactivity.
Sporadic serotonin-positive cells were observed in the epidermis of the fins both in the stump and in the regenerate (Figure 1B' and E').

Taken together, at $3 \mathrm{dpa}$, serotonin is detected in the basalwound epithelium and mesenchyme in two opposite gradients along the proximo-distal axis (Figure 2C). In the mesenchyme, the highest levels are present in the proximal blastema and the lowest in the distal-most blastema, whereas, in the wound epithelium, the strongest expression was at the distal-most position and the weakest one in the proximal region.

\section{Expression of serotonin-synthetizing enzymes in the regenerating fins}

The first step of serotonin synthesis involves the conversion of the amino acid L-tryptophan by the enzyme tryptophan hydroxylase (Tph). ${ }^{12,13}$ To determine if serotonin is produced inside the regenerating tissue, we performed qRT-PCR for all known homologous genes of tryptophan hydroxylase in zebrafish, namely tphla, tphlb, and tph2. Expression levels were detected in fins at 0 and 3 dpa relative to two different reference genes, beta-actin 2 and poly(A)-binding protein cla (pabpcla), a ubiquitously expressed gene during zebrafish development. $^{25}$ qRT-PCR indicated a two-fold increase in tphla expression and a 4-fold increase in tphlb expression between 0 and $3 \mathrm{dpa}$, while tph 2 expression remained unchanged (Figure 3A). To confirm these findings and elucidate which tissues express the different genes, we performed in situ hybridization for all three tph genes. We confirmed that tphla and tphlb were expressed in regenerating fins at low and high levels, respectively, whereas tph2 was not detected (Figure 3B-F). Consistent with previous studies, ${ }^{19,26}$ at $1 \mathrm{dpa}$, $t p h 1 b$ transcripts were strongly upregulated below the amputation plane (Figure 3B). This region corresponds to the serotonin-positive area, as detected by immunofluorescence (Figure 1C). At 2, 3, and $5 \mathrm{dpa}$, both paralogous gene transcripts, tphla and tphlb, were present in the distal blastema (Figure 3C-E). Additionally, we analyzed the expression of the monoamine oxidase (mao) gene, ${ }^{27}$ which encodes the enzyme degrading serotonin. In situ hybridization did not detect expression of this gene in the fin (Figure 3G). Overall, we observed an overlapping expression of two homologous genes of the serotonin-synthetizing enzyme tph in the regenerating fin. We concluded that the source of serotonin in the fin is the local tissue of the blastema.

\section{Neither systemic inhibition of Tph activity nor exogenous serotonin delivery affect fin regeneration}

A previous study has demonstrated that tphlb mutant fish regenerate normally. ${ }^{19}$ However, it is not known if the activity of its homologous gene tphla can compensate for the absence

Figure 3. Tryptophan hydroxylase is expressed in the regenerating fin. (A) Expression levels of tph1a, tph1b, and tph2 are determined by qRT-PCR. tph $1 a$ and tph $1 b$ are significantly upregulated at $3 \mathrm{dpa}$. $\beta$-actin 2 and pabpc $1 a$ were used as normalization. $* p<0.05 ; * * p<0.001$. Pooled data from 3 qRT-PCRs, 2 cDNAs per time point, reactions performed in duplicates. Tissue from 10 fish was used to generate each cDNA. (B-E) In situ hybridization for the serotonin synthesis enzymes tph1a and tph $1 b$ on longitudinal fin sections at $1,2,3$, and $5 \mathrm{dpa}$. Both tph $1 \mathrm{a}$ and $\operatorname{tph} 1 \mathrm{~b}$ are expressed in the distal mesenchyme of regenerating fins. (F) In situ hybridization for the serotonin synthesis enzymes tph2 on a longitudinal fin section at 3 dpa reveals the absence of this third paralogue in the regenerating fin. (G) In situ hybridization for the serotonin degrading enzymes monoamine oxidase (mao) on a longitudinal fin section at 3 dpa reveals the absence of expression in the regenerating fin. $\mathrm{N} \geq 4$ for each ISH. 


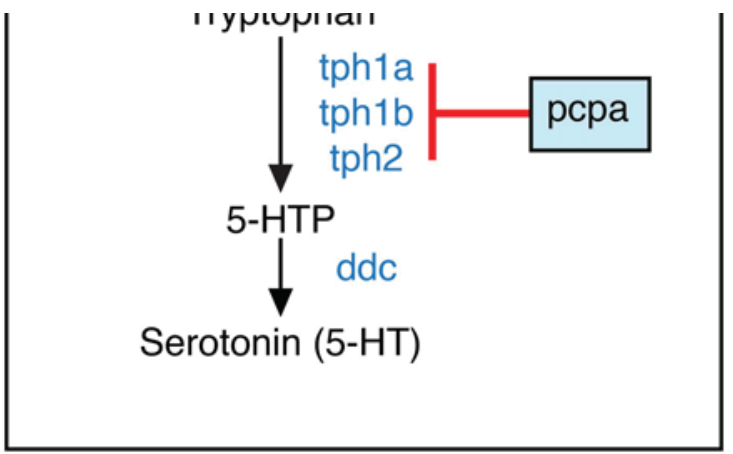

B
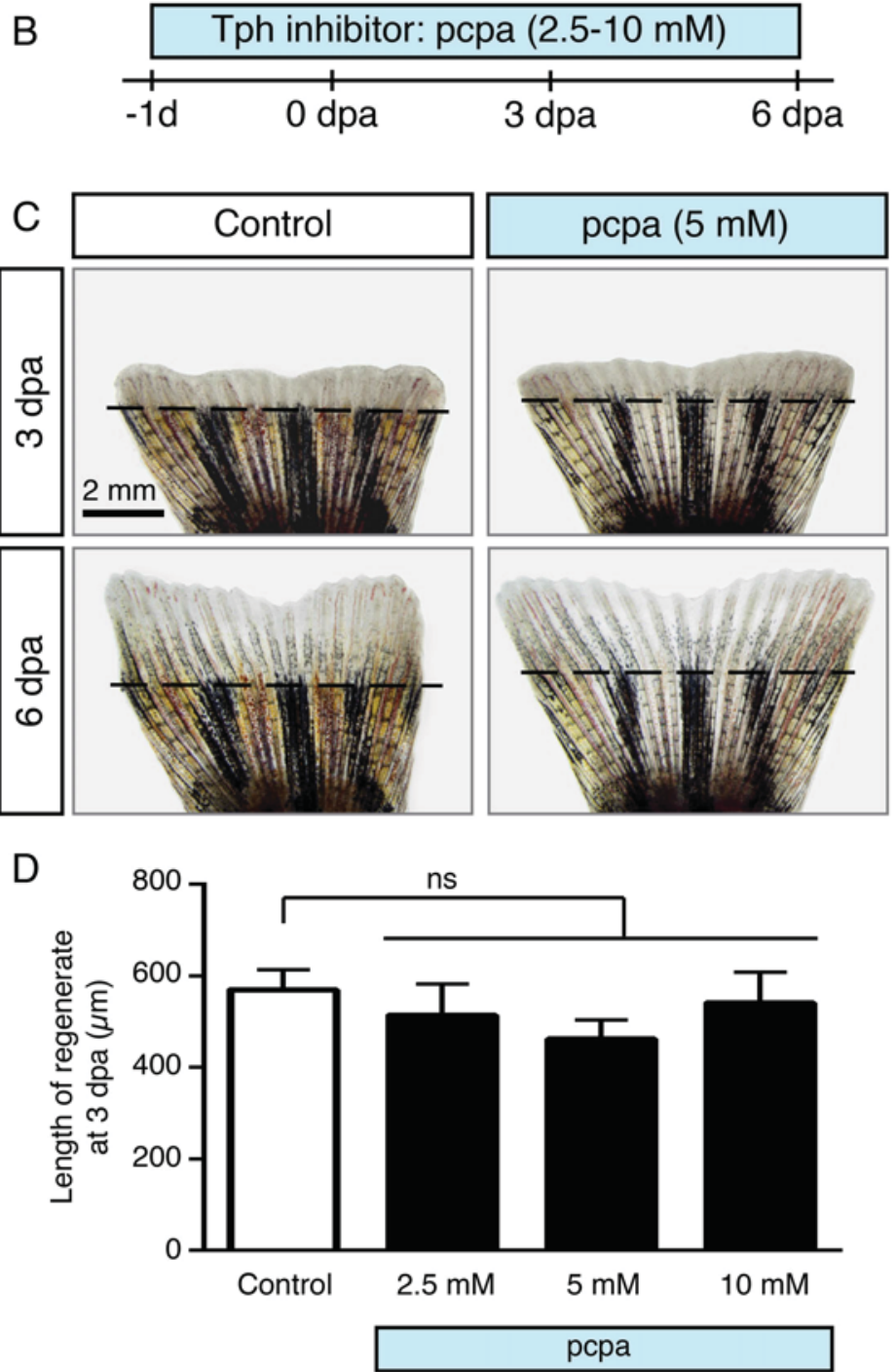
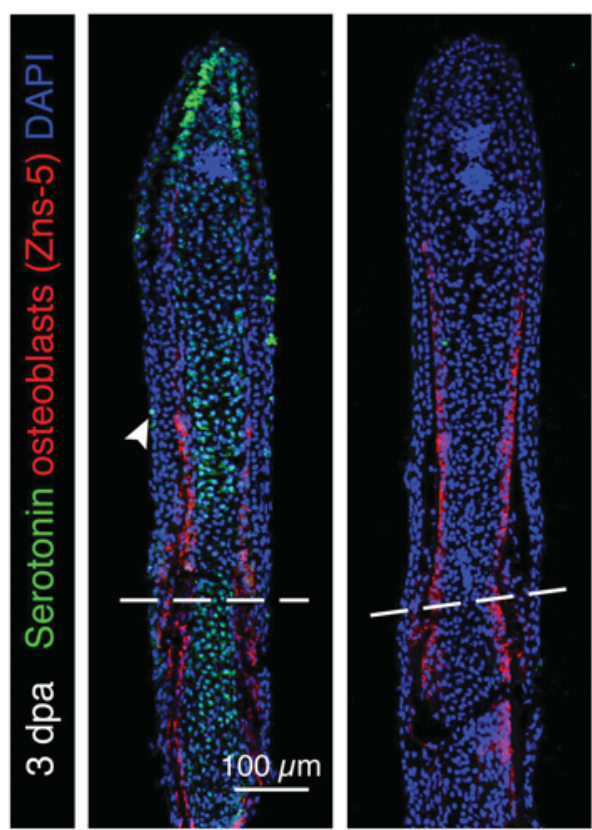

$\mathrm{F}$

$6 \mathrm{~h} \mathrm{BrdU}$
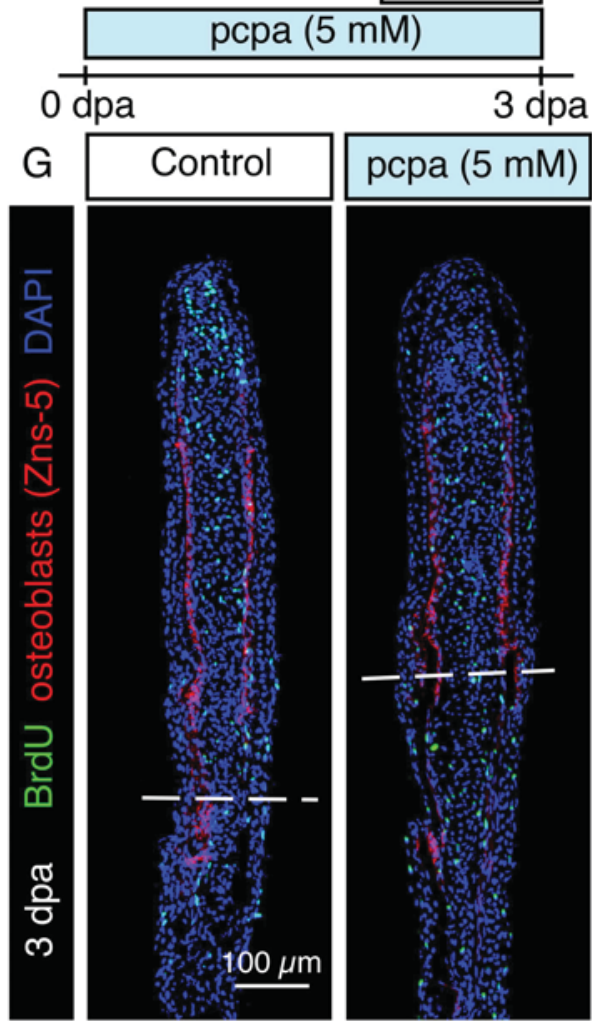

Figure 4. Inhibition of serotonin synthesis does not impair regeneration. (A) Schematic representation of the serotonin synthesis pathway, with indication of the inhibitor used in this study. (B) Experimental design for the inhibition of serotonin synthesis during regeneration using para-chlorophenylalanine (pcpa). (C) Live imaging of fins treated with pcpa and control fish at 3 and 6 dpa. (D) Quantification of length of regenerates at $3 \mathrm{dpa}$ after treatment with different concentrations of pcpa. $\mathrm{N} \geq 6$ for each group. ns = non-significant. (E) Immunofluorescence staining for serotonin and Zns-5 on 3 dpa longitudinal sections of fins treated with $5 \mathrm{mM}$ pcpa and control fins. Treatment with pcpa eliminated all serotonin from the regenerating fins in sporadic serotoninpositive epidermal cells (arrowhead), mesenchyme, and distal-most basal wound epidermis. $N \geq 3$. (F) Experimental design for BrdU incorporation assay to determine if pcpa treatment affects proliferative capacity. (G) Immunofluorescence staining for BrdU in fins treated with pcpa and control fins show similar cell proliferation. $\mathrm{N} \geq 3$ for each group. 

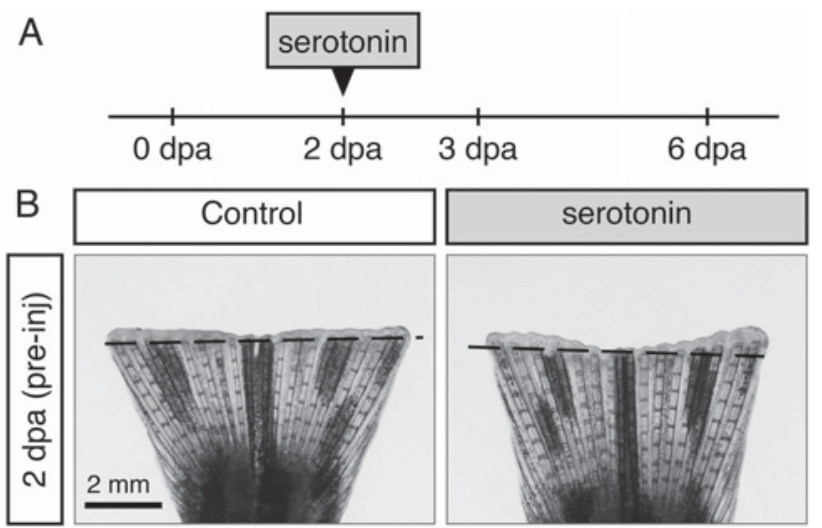

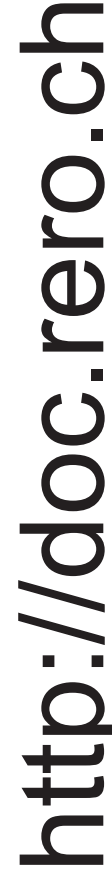

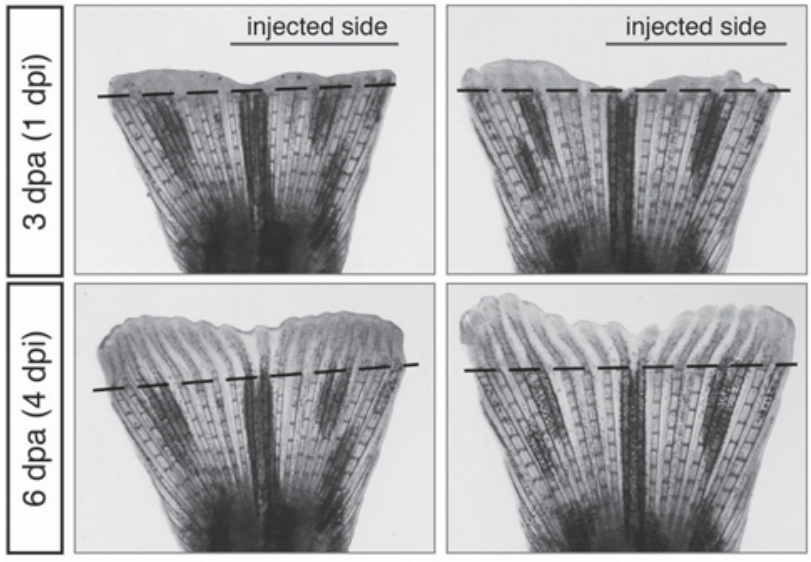

C

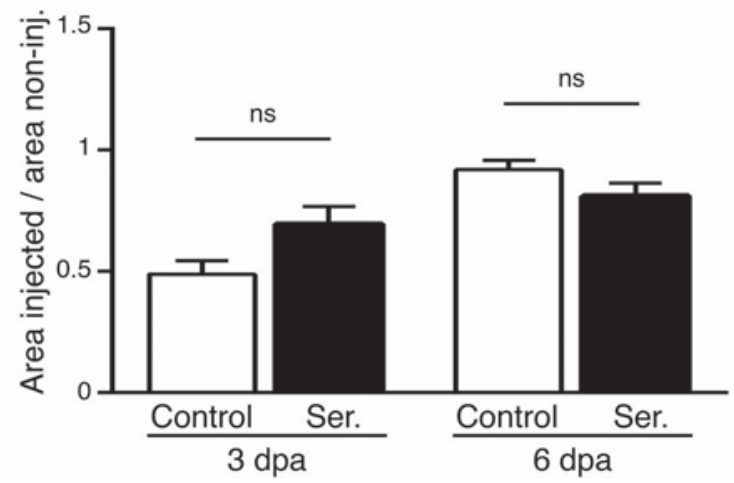

Figure 5. Serotonin injection does not affect regeneration. (A) Experimental design for injection of serotonin into regenerating blastemas. Serotonin was injected into the ventral lobe of fins at $2 \mathrm{dpa}$, corresponding to the right half of each fin image. (B) Live imaging of fins before injection, at 1 and 4 day post-injection (dpi). (C) Quantification of the effect of injections. Ratio of regenerate area of the injected lobe over the uninjected lobe. Serotonin injection did not significantly affect regeneration. $\mathrm{N} \geq 3$ fish per group.

of tphlb. To overcome this potential genetic redundancy, we systemically abrogated serotonin synthesis through treatment with a chemical Tph-antagonist, para-chlorophenylalanine (рсpa) (Figure 4A), which was previously validated in behavioral assays in zebrafish embryos and adults. ${ }^{28}$ Treatment with three different concentrations of this drug $(2.5,5$ and $10 \mathrm{mM})$ for 1 day before amputation and up to 6 days postamputation did not affect fin regeneration as seen on live imaging of the fins and through quantification of the regenerate length (Figure 4B-D). Treatment with $5 \mathrm{mM}$ pcpa was sufficient to completely abolish the serotonin immunoreactivity in the mesenchyme and wound epithelium as examined on fin sections at 3 dpa (Figure 4E). Despite the complete absence of serotonin, we did not observe any change in cell proliferation in the regenerating fin, as analyzed by a BrdU incorporation assay (Figure $4 \mathrm{~F}-\mathrm{G}$ ).

To test whether exogenous serotonin can modulate regeneration, we injected $0.8 \mu \mathrm{g}$ of serotonin into the blastema of one lobe of fins at $2 \mathrm{dpa}$ (Figure 5A). Control fish were injected with buffer. We found that the delivery of serotonin did not alter the outgrowth size at 3 and $6 \mathrm{dpa}$, as seen on photographs of fins and by quantification of the regenerate area (Figure 5B-C). Taken together, our results indicate that neither inhibition of serotonin production nor local injection of serotonin affected regeneration of caudal fins in zebrafish.

\section{FGF signaling is required for synthesis of serotonin in the regenerating fin}

Various signaling pathways have been implicated in the induction of regeneration in the zebrafish fin. To investigate which of these pathways trigger serotonin synthesis in the regenerating fin, we used a combination of mutant and transgenic fish, as well as pharmacological inhibitors to interfere with certain pathway activities.

Fibroblast growth factor (FGF) signaling is a key factor for fin regeneration. ${ }^{20,21,29}$ To investigate the role of the FGF pathway in serotonin production during regeneration, we used fgf $20 a$ homozygous mutant fish called devoid-ofblastema $(d o b)$, which fail to regenerate fins. ${ }^{21}$ We found that fgf2Oa mutant fins lacked serotonin and tphla/b expression in the mesenchyme at $3 \mathrm{dpa}$ (Figure 6B and C). Remarkably, sporadic serotonin-positive cells of the epidermis were not affected in $f g f 20 a$ mutant fins. Thus, $f g f 20 a$ is necessary for the expression of serotonin-producing enzymes and serotonin accumulation in the blastema and basal wound epithelium during regeneration.

To further determine the role of FGF in serotonin accumulation, we applied a genetic approach by using the transgenic fish $h s p 70:$ dnfgfrl-egfp, which overexpress a dominant negative form of the FGF receptor type 1 after heat shock induction. $^{20}$ The heat shock was induced at $2 \mathrm{dpa}$, after the establishment of the normal blastema (Figure 6A). Immunofluorescence staining of fin sections at $3 \mathrm{dpa}$, showed no serotonin in the regenerative outgrowth (Figure 6B). However, the expression of the serotonin-synthetizing genes tphla and $t p h l b$ was preserved in the regenerative outgrowth (Figure 6C). These results suggest that FGF signaling is not required for maintenance of the tphla/b expression in the blastema. Similar results were observed after pulse treatment with $1 \mu \mathrm{M}$ retinoic acid (RA) for 1 day starting at 2 dpa (Figure 6D-F). The expression of the tphlb enzyme was enhanced within the entire blastema, but serotonin accumulation in the same region was suppressed. We concluded that cytoplasmic serotonin accumulation is not merely dependent on the expression of the ratelimiting enzyme but involves downstream mechanisms.

Finally, we assessed deposition of serotonin after inhibition of another pathway, TGF $\beta /$ Activin- $\beta \mathrm{A}$, which is also essential 


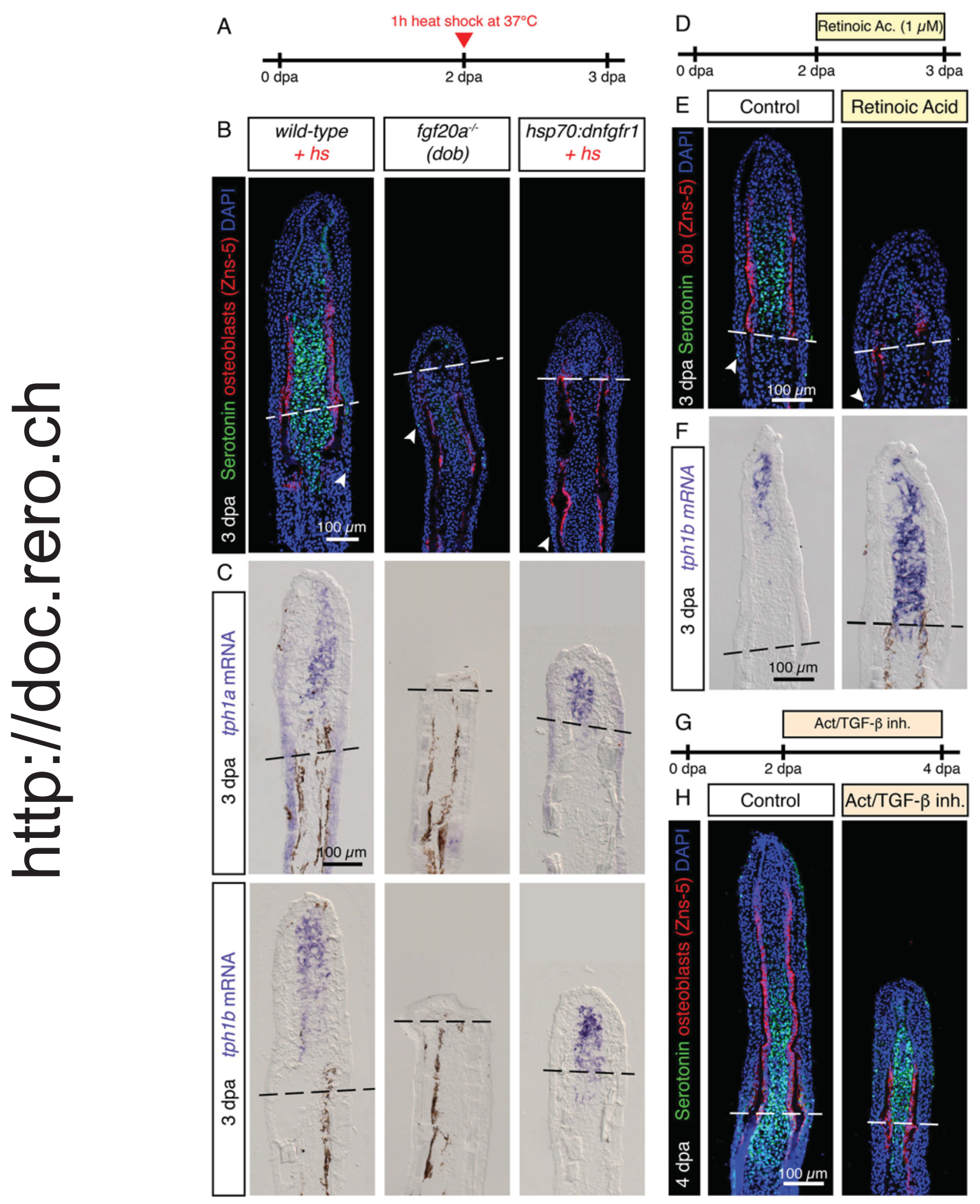

Figure 6. Legend on next page. 
for regeneration, but most likely acts in parallel to FGF signaling. ${ }^{5}$ The treatment of fins with $10 \mu \mathrm{M}$ SB431542, a validated TGF $\beta /$ Activin- $\beta$ A inhibitor, ${ }^{30-32}$ for 2 days starting from 2 dpa impaired progression of regeneration without affecting serotonin in the blastema (Figure $6 \mathrm{G}$ and $\mathrm{H}$ ). Thus, inhibition of regeneration in an FGF-independent manner does not affect serotonin expression, and we, therefore, conclude that the absence of serotonin in the blastema after interference with FGF and retinoic acid signaling is pathway specific.

\section{DISCUSSION}

Natural appendage regeneration in lower vertebrates, such as zebrafish and salamanders, predominantly relies on the intrinsic plasticity of mature tissues. This property allows local activation of the stump below the amputation plane to promote cell division, migration, and complete reproduction of the missing structure. The elucidation of the molecular mechanisms underlying this process is of critical importance in the field of regenerative biology. Here, we identified that the activation of mesenchymal cells of the stump after an amputation involves a transient serotonin deposition. Consistent with a previous study, ${ }^{19}$ the regenerative outgrowth contained serotonin in the proximal blastema and in the distal wound epithelium. Our detailed immunofluorescence analysis revealed that the wound epithelium and the mesenchyme displayed two inverse concentration gradients of cellular serotonin with the strongest levels in the distal epithelium and the proximal mesenchyme (Figure 2C). Remarkably, the serotonin-positive blastema remained associated with the amputation area up to $5 \mathrm{dpa}$, despite the elongation of the outgrowth.

The annotated zebrafish genome contains three genes encoding the serotonin synthesizing enzymes, tphla, tphlb, and tph2. Using qRT-PCR and in situ hybridization, we demonstrated that two paralogs, tphla and tphlb, are expressed in the blastema of regenerating fins. A comparison of the distribution between enzyme transcripts and serotonin revealed a colocalization after wound healing at $1 \mathrm{dpa}$. However, during outgrowth formation, at $3 \mathrm{dpa}$, a discrepancy in location can be observed: the highest expression of tphlalb was located in the distal blastema, while serotonin remained near the amputation plane. This mismatch suggests the existence of metabolic differences between tissues at the original injury site and tissues at the protruding outgrowth. In this scenario, the injury/wound healing tissue would create a biosynthetic and extracellular environment, which would favor the retention of serotonin. During outgrowth formation, the protruding distal blastema would move out of reach of the original injury/wound healing signals. Far from the injury, distal blastemal cells would adjust their metabolism to new conditions, which do not support serotonin retention. This hypothesis would be consistent with the current knowledge about the compartmentalization of the blastema into its proximal and distal parts starting at approximately $3 \mathrm{dpa} .{ }^{1,6}$ These subregions display different proliferative patterns, differentiation levels, and gene expressions. The subdivision of the blastema is maintained by the interplay of various signaling pathways, such as Wnt, FGF, retinoic acid, and Notch. ${ }^{\top}$ Here, we have shown that the FGF and retinoic acid signaling pathways contribute to the serotonin accumulation. Our study demonstrates that serotonin represents a novel marker of the injury/wound healing zone, which persists at the base of the outgrowth during a few days after initiation of regeneration.

At the time point of blastema compartmentalization at $3 \mathrm{dpa}$, another peculiarity was observed: the basal layer of the wound epithelium became positive for serotonin in the region covering the distal-most serotonin-negative blastema. It is possible that the distal-most wound epithelium uptakes serotonin from the distal blastema. This hypothesis is consistent with the model of serotonin transport across the tissues from the mesenchyme up to epidermis. ${ }^{33,34}$ Further studies are required to elucidate further molecular mechanisms regulating the distribution of serotonin in the regenerating fin.

The significance of the transient accumulation of serotonin in the proximal blastema remains mysterious. A previous study demonstrated that $t p h 1 b$ mutant fish regenerate fins normally. ${ }^{19}$ This could be explained by compensation by the paralogous gene, tphla, which is also expressed during fin regeneration. However, our experiment with the pharmacological inhibitor of Tph, parachlorophenylalanine, confirmed that serotonin is not required for fin regrowth. Furthermore, injection of ectopic serotonin into fin regenerates did not modulate regeneration. Serotonin might be a temporal side product of the metabolic change, which occurs when the tissue switches from the healing to regeneration response. Nevertheless, the retained cytoplasmic serotonin provides a new marker for mesenchymal cells at the injury/wound healing zone of the regenerating fin. Understanding the regulation of serotonin and its biological implication will provide a better understanding of regenerative plasticity in vertebrate appendages.

\section{ACKNOWLEDGMENT}

We are grateful to V. Zimmermann for technical help and animal care, C. Pfefferli for critical reading of the manuscript.

\footnotetext{
Figure 6. Serotonin production depends on fibroblast growth factor and retinoic acid signaling. (A) Experimental design for the investigation of the role of the FGF pathway for the production of serotonin. Wild-type, fgf20a ${ }^{-/}$, and hsp70:dnfgfr 1-egfp fish were used. (B) Immunofluorescence staining for serotonin and Zns-5 in wild-type, fgf20a ${ }^{-/}$and hsp70:dnfgfr 1 fish at 3 dpa. Mesenchymal serotonin was absent in fish with inactive FGF signaling. Serotonin in sporadic epidermal cells (arrowheads) was not affected. $\mathrm{N} \geq 4$ fish of each genotype. (C) In situ hybridization for tph1a and tph1b in wild-type, fgf20a ${ }^{-/}$and hsp70:dnfgfr1-egfp fish at 3 dpa. tph $1 a$ and tph $1 b$ were absent in $\mathrm{fgf}_{20 a^{--}}$fish, whereas they were maintained in hsp70:dnfgfr 1 fish. $\mathrm{N} \geq 4$ fish of each genotype. (D) Experimental design for the investigation of the effect of retinoic acid (RA) treatment on serotonin production. (E) Immunofluorescence staining for serotonin in RA treated and control fins at $3 \mathrm{dpa}$. Mesenchymal serotonin was absent after RA treatment. Serotonin in sporadic epidermal cells (arrowheads) was not affected. $N=3$ fish per group. (F) In situ hybridization for tph $1 b$ in RA-treated fins and control at $3 \mathrm{dpa}$. tph $1 \mathrm{~b}$ was strongly upregulated after RA treatment. $N=3$ fish per group (G) Experimental design for the investigation of the effect of TGF- $\beta / A c t i v i n \beta A$ signaling inhibition using the inhibitor $10 \mu \mathrm{M}$ SB431542. (H) Immunofluorescence staining for serotonin in SB431542 treated and control fins at 4 dpa. Serotonin was not affected by the inhibition of TGF- $\beta /$ Activin $\beta A$ signaling. $N=3$ fish per group.
} 
Source of Funding: This work was supported by the Swiss National Science Foundation, grant numbers: 310030_ 179213 and CRSII3_147675.

Conflict of Interest: The authors declare no financial or non-financial competing interests.

\section{REFERENCES}

1. Tornini VA, Poss KD. Keeping at arm's length during regeneration. Dev Cell 2014; 29: 139-45.

2. Jazwinska A, Sallin P. Regeneration versus scarring in vertebrate appendages and heart. J Pathol 2016; 238: 233-46.

3. Sousa S, Afonso N, Bensimon-Brito A, Fonseca M, Simoes M, Leon J, et al. Differentiated skeletal cells contribute to blastema formation during zebrafish fin regeneration. Development 2011; 138: 3897-905.

4. Knopf F, Hammond C, Chekuru A, Kurth T, Hans S, Weber $\mathrm{CW}$, et al. Bone regenerates via dedifferentiation of osteoblasts in the zebrafish fin. Dev Cell 2011; 20: 713-24.

5. Pfefferli C, Jazwinska A. The careg element reveals a common regulation of regeneration in the zebrafish myocardium and fin. Nat Commun 2017; 8: 15151.

6. Pfefferli C, Jaźwińska A. The art of fin regeneration in zebrafish. Regeneration 2015; 2: 72-83.

7. Wehner D, Weidinger G. Signaling networks organizing regenerative growth of the zebrafish fin. Trends Genet 2015; 31: 336-43.

8. Petrie TA, Strand NS, Yang CT, Rabinowitz JS, Moon RT. Macrophages modulate adult zebrafish tail fin regeneration. Development 2014; 141: 2581-91.

9. Simões MG, Bensimon-Brito A, Fonseca M, Farinho A, Valério $F$, Sousa $S$, et al. Denervation impairs regeneration of amputated zebrafish fins. BMC Dev Biol 2014; 14: 49.

10. Canli T, Lesch K-P. Long story short: the serotonin transporter in emotion regulation and social cognition. Nat Neurosci 2007; 10: 1103-9.

11. Jonnakuty C, Gragnoli C. What do we know about serotonin? J Cell Physiol 2008; 217: 301-6.

12. Lv J, Liu F. The role of serotonin beyond the central nervous system during embryogenesis. Front Cell Neurosci 2017; 11: 74 .

13. Amireault P, Sibon D, Côté F. Life without peripheral serotonin: insights from tryptophan hydroxylase 1 knockout mice reveal the existence of paracrine/autocrine serotonergic networks. ACS Chem Nerosci 2013; 4: 64-71.

14. Martin AM, Young RL, Leong L, Rogers GB, Spencer NJ, Jessup CF, et al. The diverse metabolic roles of peripheral serotonin. Endocrinology 2017; 158: 1049-63.

15. Herculano AM, Maximino C. Serotonergic modulation of zebrafish behavior: towards a paradox. Prog Neuro-Psychopharmacology Biolog Psychiat 2014; 55: 50-66.

16. Coccimiglio ML, Jonz MG. Serotonergic neuroepithelial cells of the skin in developing zebrafish: morphology, innervation and oxygen-sensitive properties. J Exp Biol 2012; 215: 3881-94.

17. Zachar PC, Jonz MG. Confocal imaging of Merkel-like basal cells in the taste buds of zebrafish. Acta Histochem 2012; 114: 101-15.

18. Soulika M, Kaushik A-L, Mathieu B, Lourenço R, Komisarczuk AZ, Romano SA, et al. Diversity in cell motility reveals the dynamic nature of the formation of zebrafish taste sensory organs. Development 2016; 143: 2012-24.
19. Tornini VA, Thompson JD, Allen RL, Poss KD. Live fatemapping of joint-associated fibroblasts visualizes expansion of cell contributions during zebrafish fin regeneration. Development 2017; 144: 2889-95.

20. Lee Y, Grill S, Sanchez A, Murphy-Ryan M, Poss KD. Fgf signaling instructs position-dependent growth rate during zebrafish fin regeneration. Development 2005; 132: 5173-83.

21. Whitehead GG, Makino S, Lien CL, Keating MT. fgf20 is essential for initiating zebrafish fin regeneration. Science 2005; 310: $1957-60$

22. König D, Page L, Chassot B, Jaźwińska A. Dynamics of actinotrichia regeneration in the adult zebrafish fin. Dev Biol ; 2018; 433: 416-432.

23. Montgomery JE, Wiggin TD, Rivera-Perez LM, Lillesaar C, Masino MA. Intraspinal serotonergic neurons consist of two, temporally distinct populations in developing zebrafish. Dev Neurobiol 2016; 76: 673-87.

24. Johnson SL, Weston JA. Temperature-sensitive mutations that cause stage-specific defects in zebrafish fin regeneration. Genetics 1995; 141: 1583-95.

25. Mishima Y, Fukao A, Kishimoto T, Sakamoto H, Fujiwara T, Inoue K. Translational inhibition by deadenylation-independent mechanisms is central to microRNA-mediated silencing in zebrafish. Proc Natl Acad Sci 2012; 109: 1104-9.

26. Tornini VA, Puliafito A, Slota LA, Thompson JD, Nachtrab G, Kaushik AL, et al. Live monitoring of blastemal cell contributions during appendage regeneration. Curr Biol 2016; 26: 2981-91.

27. Maximino C, Gomes M, Araujo J, Oliveira K, Herculano A, Stewart A, et al. The serotonergic system of zebrafish: genomics, neuroanatomy and neuropharmacology. In: Hall S , editor Serotonin: Biosynthesis, Regulation and Health Implications. New-York, United-States: Nova Science, 2013; 53-67.

28. Maximino C, Puty B, Benzecry R, Araújo J, Lima MG, de Jesus Oliveira Batista E, et al. Role of serotonin in zebrafish (Danio rerio) anxiety: relationship with serotonin levels and effect of buspirone, WAY 100635, SB 224289, fluoxetine and Para-chlorophenylalanine (pCPA) in two behavioral models. Neuropharmacology 2013; 71: 83-97.

29. Poss KD, Shen J, Nechiporuk A, McMahon G, Thisse B, Thisse C, et al. Roles for Fgf signaling during zebrafish fin regeneration. Dev Biol 2000; 222: 347-58.

30. Jazwinska A, Badakov R, Keating MT. Activin-betaA signaling is required for zebrafish fin regeneration. Curr Biol 2007; 17: $1390-5$.

31. Chablais F, Jazwinska A. The regenerative capacity of the zebrafish heart is dependent on TGFbeta signaling. Development 2012; 139: 1921-30.

32. Marro J, Pfefferli C, de Preux Charles AS, Bise T, Jazwinska A. Collagen XII contributes to epicardial and connective tissues in the zebrafish heart during ontogenesis and regeneration. PLoS One 2016; 11: e0165497.

33. Moiseiwitsch J, Lambert H, Lauder J. Roles for serotonin in non-neural embryonic development. In: Kalverboer AF, Gramsbergen A, editors. Handbook of Brain and Behavior in Human Development. Great Britain: Kluwer Academic Publishers, 2001; 139-152.

34. Fukumoto T, Kema IP, Levin M. Serotonin signaling is a very early step in patterning of the left-right Axis in Chick and Frog embryos. Curr Biol 2005; 15: 794-803. 